\title{
Generalized earthquake classification
}

\author{
Ben Lasscock ${ }^{\ddagger *}$ \\ https://youtu.be/uT0Nkf0BA7o
}

\begin{abstract}
We characterize the source of an earthquake based on identifying the nodal lines of the radiation pattern it produces. These characteristics are the mode of failure of the rock (shear or tensile), the orientation of the fault plane and direction of slip. We will also derive a correlation coefficient comparing the source mechanisms of different earthquakes. The problem is formulated in terms of a simple binary classification on the surface of the sphere. Our design goal was to derive an algorithm that would be both robust to misclassification of the observed data and suitable for online processing. We will then go on to derive a mapping which translates the learned solution for the separating hyper-plane back to the physics of the problem, that is, the probable source type and orientation. For reproducibility, we will demonstrate our algorithm using the example data provided with the HASH earthquake classification software, which is available online.
\end{abstract}

Index Terms—machine learning, earthquake, hazard, classification.

\section{Introduction}

In this paper we are going to explain how to classify earthquake data using a support vector classifier (SVC) and then how to interpret the result physically. We will be drawing on the scikitlearn [sklearn] project for the SVC, the ObsPy seismological Python package [ObsPy] for some utility routines and mplstereonet [mplstereonet], which is a matplotlib [mpl] plugin for visualization.

Much of the discussion will center around deriving a mapping from the solution of the SVC to the physical process that originated the earthquake. The key concept we will be elaborating on is understanding the relationship between what we call the input and feature spaces of the SVC. The results of the classification are curves separating points on the surface of the focal sphere (the input space), which is the domain of the input data. However, the physics and understanding of the result lies in the representation of the solution in the feature space, which a higher dimensional space where the classifier may linearly separate the data.

For the sake of reproducibility, the demonstration will use the same dataset provided with the US Geological Survey (USGS) HASH software. HASH [HASH] is an earthquake classification code provided by the USGS and it is built upon an earlier package called FPFIT, which implements a least squares classifier. For each case we will be comparing and contrasting our solutions with those generated by HASH, which we generally expect to be similar.

* Corresponding author: blasscoc@gmail.com

\$ Geotrace Technologies

Copyright $(2016$ Ben Lasscock. This is an open-access article distributed under the terms of the Creative Commons Attribution License, which permits unrestricted use, distribution, and reproduction in any medium, provided the original author and source are credited.

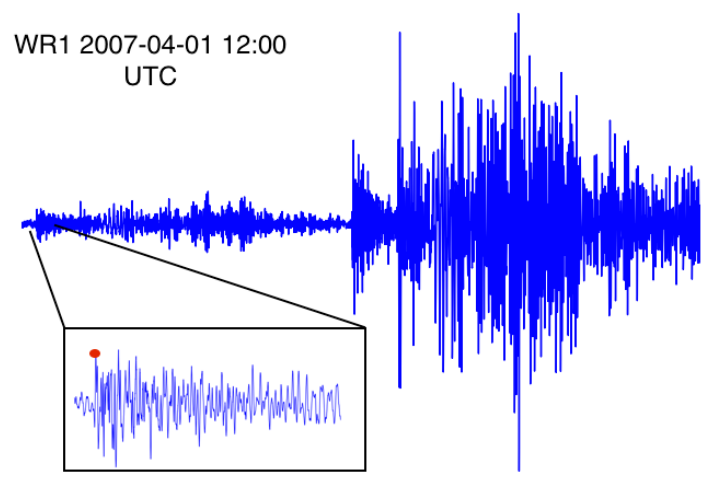

Fig. 1: A seismogram measured at the WR1 node of the Warramunga seismic array showing displacement due to an aftershock of the April 2007 Solomon Islands earthquake. The red dot indicates the first break motion. The data was obtained by querying the IRIS database http: //ds.iris.edu/ds/nodes/dmc/data/types/events/.

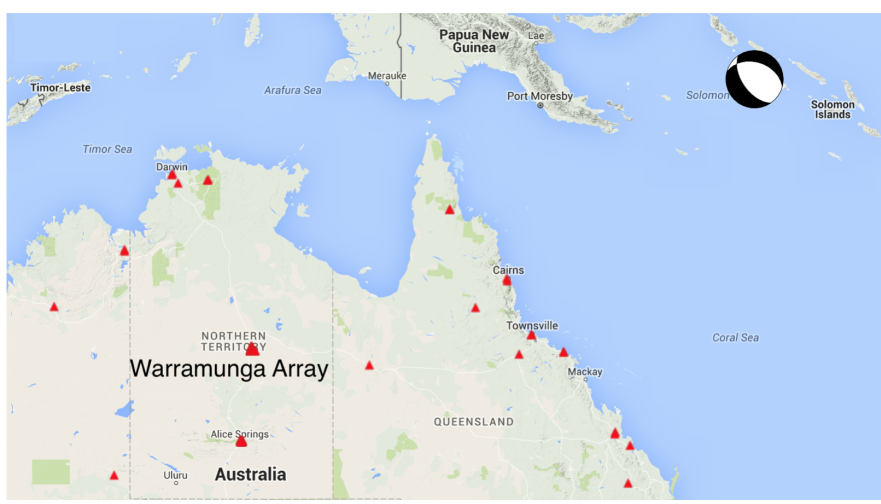

Fig. 2: A portion of the Australian seismic network showing the location of the Warramunga seismic array, the map was obtained from http://www.fdsn.org/networks/detail/AU/.

Our discussion will include annotated software explaining the important steps in the computation. We will be contributing software $^{1}$ to reproduce the results of this paper.

1. https://github.com/blasscoc/FocalMechClassifier 


\section{Problem statement}

Consider a particular example where we would apply the analysis presented in this paper. In Fig. 1, we show a seismogram (recording) of an earthquake that was located in Solomon Islands. The black and white glyph is a graphical representation of the type of focal mechanism. The orientation of the nodal lines of this glyph displays the orientation of the fault plane. The recording shown was made by the WR1 node of the Warramunga seismic array, which is part of the Australian seismic network, shown partially in Fig. 2. The goal of hazard monitoring in this case would be to characterize the deformation of the earth (focal mechanism) that caused this earthquake. This analysis would involve first locating the source spatially and then classifying its focal mechanism, and importantly the orientation of the fault plane. The orientation of the fault plan is important because displacement of the sea-floor can cause the formation of tsunamis. The algorithm discussed in this paper provides the analysis of the focal mechanism, with the extension that we can also compare the spectrum of the solution with a past events located in this area. This additional information may be useful for decision making regarding what action should be taken given the risk of a historical (or perhaps modeled) scenario repeating.

We proceed by detailing exactly the parameters of the problem at hand. The raw data are recordings of the initial arrival of energy from this earthquake, measured across a seismic network. From each recording, the initial displacement (or first motion) is identified (or picked), as shown by a red dot in Fig. 2. Consider this is as a radiation amplitude from the earthquake measured at a particular location. Further measurements across the seismic network begin to inform the shape of the radiation pattern created by the event. However, a radiation pattern measured far from the event becomes distorted because of the refraction of the seismic wave as it propagates through the earth. To remove this distortion, this energy must migrated, along an estimated ray path, back to the neighborhood of the estimated source location. We call this neighborhood the focal sphere. The process of picking, locating and migrating seismic events is beyond the scope of this paper. However, seismograms can be requested from the IRIS database ${ }^{2}$ and a suite of Python tools for processing this data is made available by the ObsPy [ObsPy] Python project.

The input data to our analysis is the polarity (signed amplitude) of the picks, and the azimuth and co-latitude of the observation migrated onto the focal sphere.The design goal is to provide an online tool for characterizing the source mechanism. The emphasis is on robustness of the algorithm, without the need for post facto processing of the data. We also need a system that provides natural metrics of similarity between seismic events.

Physically, the initial arrival of energy will be in the form of a compressional wave. The amplitude of these compressional waves are solutions to the scalar wave equation, which are the spherical harmonic functions ${ }^{3}$. Hence any function that classifies the polarity data should be a superposition of these spherical harmonics. We will learn this classifying function using the SVC. However, it is the spectral representation (harmonic content) of the radiation pattern that contains the physical meaning of the solution.

\footnotetext{
2. http://www.iris.edu

3. http://docs.scipy.org/doc/scipy/reference/generated/scipy.special.sph_ harm.html
}

\begin{tabular}{ccc} 
Source & (Fault normal/slip) & Template \\
\hline Shear & $(31)+(13)$ & $-i\left(Y_{12}+Y_{-12}\right)$ \\
Tensile & $(3)$ & $\alpha Y_{00}+4 \sqrt{5} Y_{02}$ \\
Tangential & $(3)$ & $Y_{02}-\frac{i}{2}\left(Y_{22}+Y_{-22}\right)$.
\end{tabular}

TABLE 1: Describes the angular variation of the displacement due to three types of earthquake sources in terms of a basis of spherical harmonic functions. The source templates summarized are shear, tensile and tangential dislocation. The brackets $(\cdot, \cdot)$ define the template direction of the fault normal and the direction of slip in rectangular coordinates. The constant $\alpha=2+3 \frac{\lambda}{\mu}$, where $\lambda$ and $\mu$ are the first Lamé parameter and the shear modulus respectively.

In Sec. Theory we will review the basic results we need from the theory of seismic sources. In Sec. Existing Least Squares Methods we will review existing methods for classifying earthquake data. The Sec. Earthquake - Learning with Kernels reviews the Python code used in the classification, and derives a mapping between the input space of the problem, to the feature space (represented by the spectrum). In Sec. Physical Interpretation we translate this spectral representation back to the physics of the problem, and explain how to evaluate the correlation metric. In Sec. Discussion we provide an example of the analysis and then we wrap things up with Sec. Conclusions.

\section{Theory}

The observed displacement created by the collective motion of particles along a fault plane is described by the theory of seismic sources. We will not go into all the details here, but the reference on seismic source theory we follow is Ben-Menahem and Singh [Ben81]. The key result we will draw upon is a formula for the displacement for various types of seismic sources summarized in Table 4.4 of [Ben81], which is presented in terms of Hansen vectors. Physically, a shear type failure would represent the slip of rock along the fault plane and a tensile failure would represent cracking of the rock. The results of [Ben81] are general, however we are only modeling the angular variation of the displacement due to the compressional wave measured radially to the focal sphere. From this simplification we can translate solutions of [Ben81] into solutions for just the angular variation using the basis of spherical harmonic functions, which we tabulate in Table 1. Notes on translating between [Ben81] and Table 1 are summarized in the Appendix. This result gives us an analytical expression for the spectral content of seismic sources given a certain orientation of the fault plane. We will use this information to find general solutions in Sec. Physical Interpretation.

The amplitude of the radiation pattern cannot typically be migrated back to the location of the event unless an accurate model of seismic attenuation is available, which is not generally the case, even in commercial applications. However, supposing the source type and orientation were known, then the sign of this radiation pattern is a function that must classify the polarity data on the focal sphere. As an example, in Fig. 3 we render in, 3-dimensions, the signed radiation pattern predicted for shear and tensile source, in a particular orientation.

The black areas of this beachball diagram represents the region where the displacement at the source is radially outward (vice versa for the white regions). The nodal lines represent the separating margin between classes of data (outward and inward displacement). For the shear source, the nodal lines are called the fault and auxiliary planes respectively. 


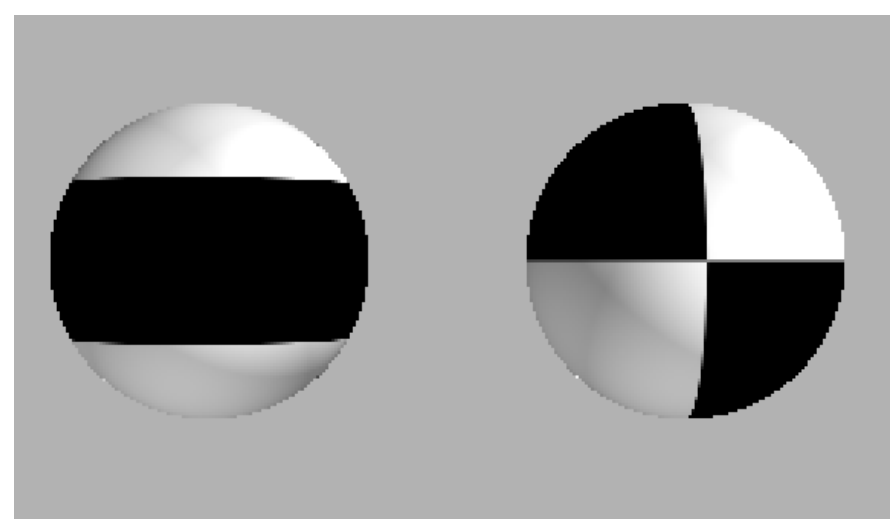

Fig. 3: Rendered in 3-dimensions, (left) the signed radiation pattern for a possible tensile type source. (right) Similarly for the case of shear type source. Figures are generated using SciPy's spherical harmonic functions and Mayavi.

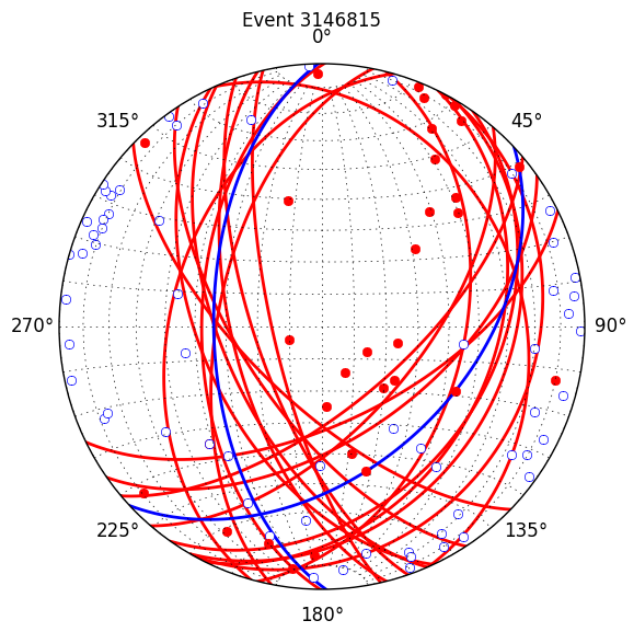

Fig. 4: For event 3146815 from north1 dataset (blue) preferred nodal line estimated by HASH, (red) a sample from the set of acceptable estimates.

One observation we can immediately take away from Fig 3 is that two diagrams are topologically different. The nodal lines of the shear source are great circles, which is not the case from the tensile source. That means there is no rotation or smooth deformation that can make one look like the other. This suggests that the two source are distinguishable, but also that there is some potential of identifying admixtures of the two based on their spectral content.

\section{Existing Least Squares Methods}

Currently, a common method (called FPFIT [FPFIT]) for earthquake classification is to assume that shear failure is the source mechanism, and then, through a least squares optimization, find the fault plane orientation that minimizes the rate of misclassification to the data. A modern code built upon FPFIT is the HASH algorithm [HASH]. The HASH software is available for download from the USGS $^{4}$ website. The HASH software comes with an example "NorthRidge" dataset which we will use to demonstrate our method. We compare the results of our algorithm with the results of HASH, which is the current state of the art. HashPy

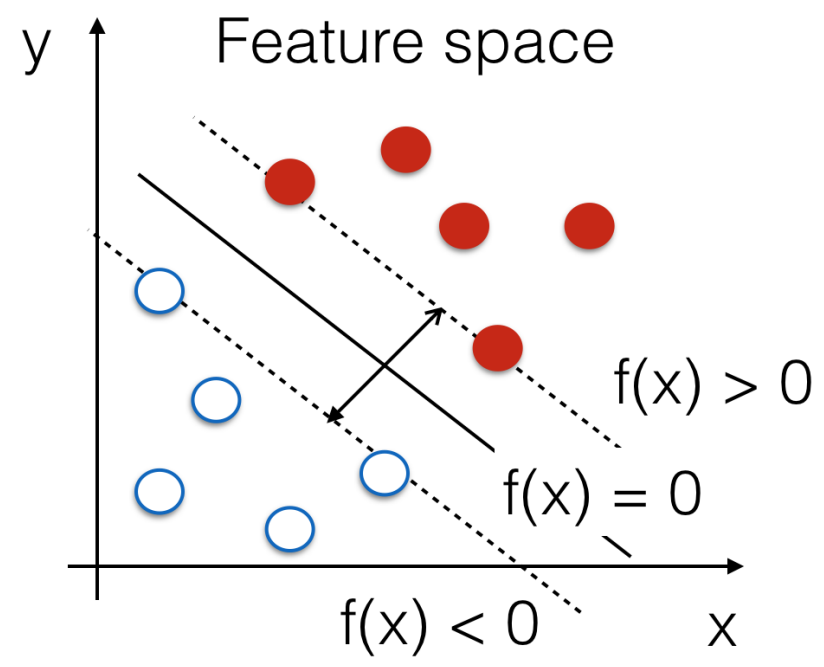

Fig. 5: A schematic of the optimization strategy of the SVC. The dashed lines represent the edges of the separating margin. The blue open and red closed dots are the polarity data represented in a feature space. The dashed lines represent a separating margin between the two classes, the solid line represents the optimal separating hyperplane.

[HashPy] is a Python project for that provides a wrapper for HASH.

Figure 4 demonstrates how the FPFIT algorithm works. The coordinate system in the figure is a stereonet projection [mplstereonet] of the lower half space of a sphere. The solid red (open blue) dots are positive (negative) polarity measured across a seismic network for the 3146815 event, which was taken from the Northridge dataset "north1.phase" supplied with the HASH software. Recall, FPFIT is a least squares method, however the function it is optimizing need not be convex. As such, there are many solutions that have a similar goodness of fit. Using a grid search method, FPFIT draws a ensemble of these possible solutions (red lines). The blue line is the preferred or most likely solution.

\section{Earthquake - Learning with Kernels}

In this section we discuss the classification algorithm we develop using the scikit-learn [sklearn] library. Whilst our interest was classification of earthquakes, the algorithm is applicable for any classification problem defined on a sphere.

Define the input space of the problem as the surface of the focal sphere, represented for example by the stereonet in Fig. 4. The data is not linearly separable on this space. The strategy of the SVC is to project the problem into a higher dimensional feature space. And in this feature space, determine the best hyperplane to separate the two classes of data by maximizing the width of the separating margin, subject to the constraint that the classes are either side of the separating margin, Fig. 5 shows a schematic of the algorithm. An important feature of the SVC is that it is robust to misclassification close to the decision boundary. Physically these are curves where the amplitude of the radiation is becoming small and then changing sign. What we believe to

4. http://earthquake.usgs.gov/research/software/index.php 
be more important than the overall rate of misclassification of the algorithm, is the stability of the result given erroneous input data.

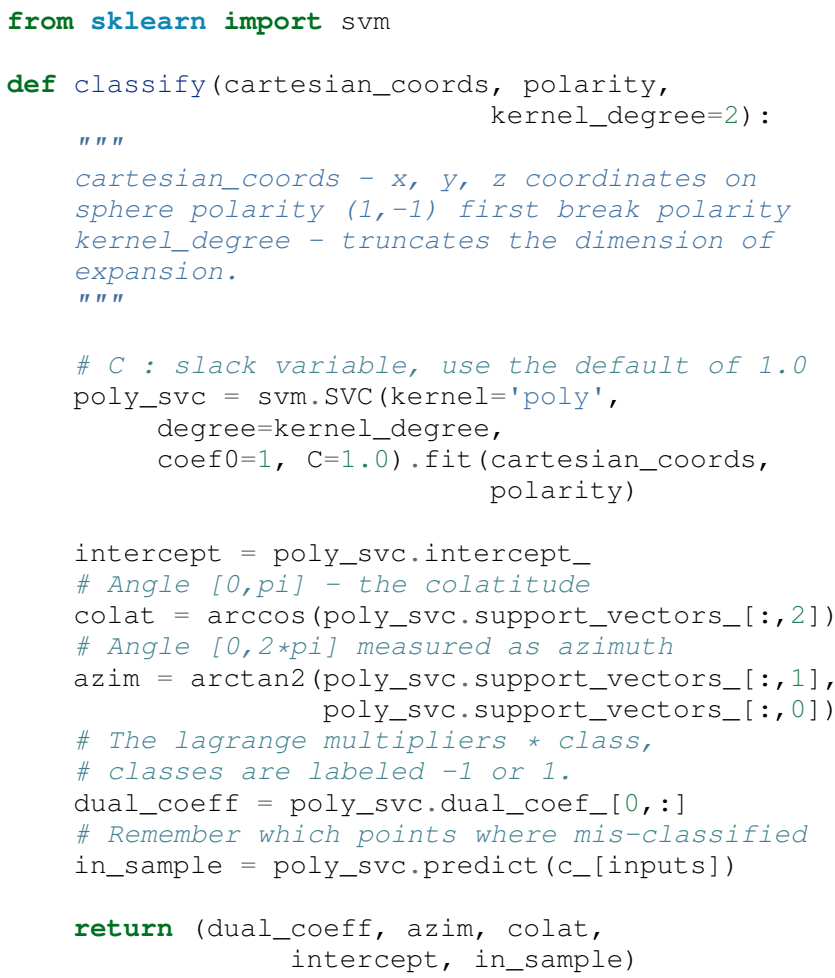

A Python implementation of the support vector classifier ${ }^{5}$ is included in scikit-learn. The projection to a higher dimensional space is done using a kernel, and evaluated in the input space using the kernel trick. For classification on a sphere, we need to use an inner product kernel, which has the form

$$
k\left(\vec{x}, \vec{x}_{i}\right)=\left(\left\langle\vec{x}, \vec{x}_{i}\right\rangle+1\right)^{d} .
$$

Here " $d$ " is the degree of the kernel. The parameter " $C$ " in the above code snippet is a slack variable. This provides a soft thresholding, which allows for some misclassification; the default value is usually sufficient. Given a set of data $y_{i}$, the support vector machine learns a corresponding set of coefficients $\alpha_{i}$ and intercept $\beta_{0}$, which determines a classifying function in the input space,

$$
f(\vec{x})=\sum_{i=1}^{N} \alpha_{i} y_{i} k\left(\vec{x}, \vec{x}_{i}\right)+\beta_{0} .
$$

In our application, the zero of this function is the nodal line, and the sign of the function is a prediction for the direction of the displacement radial to the focal sphere, given the observed data. Not all of the data is relevant for determining the best separating margin, many of the coefficients $\alpha_{i}$ may be zero. The support vectors are the locations of the data where $\alpha_{i}$ are non-zero. The product $\alpha_{i} y_{i}$ associated with each of the support vectors are called the dual coefficients (see the code snippet).

In Fig. 6 we demonstrate the SVC classifier applied to an event from the Northridge dataset. The red line represents zeros of the classifying function $\mathrm{f}(\mathrm{x})$, the green line is the solution for the fault (and auxiliary) planes determined by HASH. Note that the auxiliary plane is computed using the aux_plane function provided by the ObsPy library [ObsPy]. The learned nodal line is simply connected, the zeros of the classifying function $f(x)$ have been determined using matplotlib's contour function.

5. http://scikit-learn.org/stable/modules/generated/sklearn.svm.SVC.html

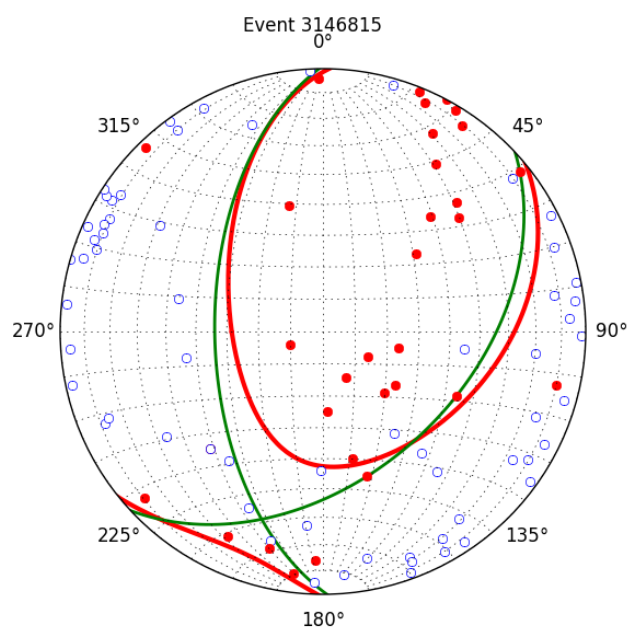

Fig. 6: For event 3146815 from the NorthRidge dataset. The green nodal line is estimated by HASH and the red nodal line is estimated by the $S V C$.

Both the HASH solution and the learned solution have a similar rate of misclassification. However the learned solution is still unsatisfactory to us because we cannot make physical sense of the result. What we want is an explanation of the type of source mechanism and its orientation. To be physically meaningful, we need an expression for the nodal lines in terms of its spectrum in the basis of spherical harmonic functions. In this basis we can then use the seismic source theory of [Ben81] to relate the result to a physical process. What we want is to determine the spectral content of $f(x)$,

$$
f(\vec{x})=\sum_{l=1}^{\infty} \sum_{m=-l}^{l} \hat{f}_{l m} Y_{l m}(\theta, \phi)
$$

that is, we want to derive its representation in the feature space. Here, the azimuth $\theta$ and colatitude $\phi$, are the angles that orientate the unit vector $\vec{x}$. The steps in deriving this representation are to first expand the inner product kernel in terms of the Legendre polynomials [Scholkopf],

$$
\begin{gathered}
a_{l}=\int_{-1}^{1} d x(x+1)^{d} P_{l}(x) \\
a_{l}=\left\{\begin{array}{ll}
\frac{2^{d+1} \Gamma(d+1)}{\Gamma(d+2+l) \Gamma(d+1-l)}+\frac{1}{2} \sqrt{\frac{1}{\pi}} \beta_{0} \delta_{l 0} & \text { if } l \leq d \\
0 & \text { otherwise }
\end{array} .\right.
\end{gathered}
$$

When we do this, we see that the degree parameter provides a natural truncation on the complexity of the function we are learning. This gives us an intermediate result which expresses the separating margin in terms of Legendre polynomials

$$
f(\vec{x})=\sum_{i=1}^{N} \alpha_{i} y_{i} \sum_{l=1}^{\infty} a_{l} P_{l}\left(\left\langle\vec{x}, \vec{x}_{i}\right\rangle\right) .
$$

The next step is to apply the addition theorem to express this in terms of the spherical harmonics,

$$
P_{l}\left(\left\langle\vec{x}, \vec{x}_{i}\right\rangle\right)=\sum_{m=-l}^{l} Y_{l m}^{*}\left(\theta^{\prime}, \phi^{\prime}\right) Y_{l m}(\theta, \phi) .
$$


The result is a formula for the spectral content of the focal mechanism given the dual coefficients estimated by the support vector classifier,

$$
\hat{f}_{l m}=\frac{4 \pi}{2 l+1} \sum_{i=1}^{N} \alpha_{i} y_{i} a_{l} Y_{l m}^{*}\left(\theta^{\prime}, \phi^{\prime}\right) .
$$

Finally, suppose we have solutions for the classification from two different sources, either observed or modeled from Table 1. A natural metric for comparing the two sources is a correlation coefficient,

$$
\rho=\frac{\|\langle g, f\rangle\|^{2}}{\|g\|\|f\|} .
$$

Using the orthogonality condition of the spherical harmonic functions, we can show that inner product is,

$$
\begin{aligned}
\langle g, f\rangle & =\int_{\infty} d^{3} x g^{*}(\vec{x}) f(\vec{x}) \\
& =\sum_{l=0}^{\infty} \sum_{m, n} \hat{g}_{l n}^{*} \hat{f}_{l m},
\end{aligned}
$$

here the integral is over the surface of the focal sphere and the star-notation means complex conjugation.

In the context of hazard monitoring, we could use the as a metric of risk, without having to propose a source mechanism or fault plane orientation.

\section{Physical Interpretation}

In the previous section we derived the general earthquake classification algorithm and a metric of correlation. Now suppose we were to assume a model for the source mechanism (e.g shear failure), how would we estimate the most likely orientation of the fault plane in this model?

First of all, in Table 1, we have a template for the spectral content of the shear source given a particular orientation. Using this template we compute a function $\mathrm{g}(\mathrm{x})$, and then generate a rotation in the input space to realign it with the classifying function $\mathrm{f}(\mathrm{x})$. This rotation would be estimated by optimizing a correlation coefficient with respect to the Euler angles,

$$
\langle g, f\rangle=\arg \max _{\alpha, \beta, \gamma} \int d^{3} x g^{*}(R(\alpha, \beta, \gamma) \vec{x}) f(\vec{x})
$$

Here, $\mathrm{R}$ represents a rotation matrix. This would be a relatively complicated procedure in the input space because we would need to re-evaluate the function $\mathrm{g}(\mathrm{x})$ at each iteration of the optimization. It is far more efficient to instead generate rotations in the feature space. To do this we borrow from quantum theory, and present Wigner's D-matrices,

$$
g(R(\alpha, \beta, \gamma) \vec{x})=\sum_{l=0}^{\infty} \sum_{m, n} D_{m n}^{l}(\alpha, \beta, \gamma) \hat{g}_{l n} Y_{l m}(\theta, \phi)
$$

Wigner's D-matrices are operators which generate rotations in the feature space of the problem. This means that we can translate a template solution (Table 1.) in a particular orientation, to a solution in any arbitrary orientation, by acting on its spectral content.

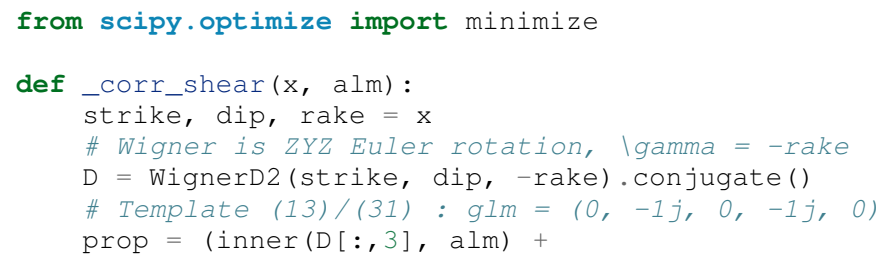

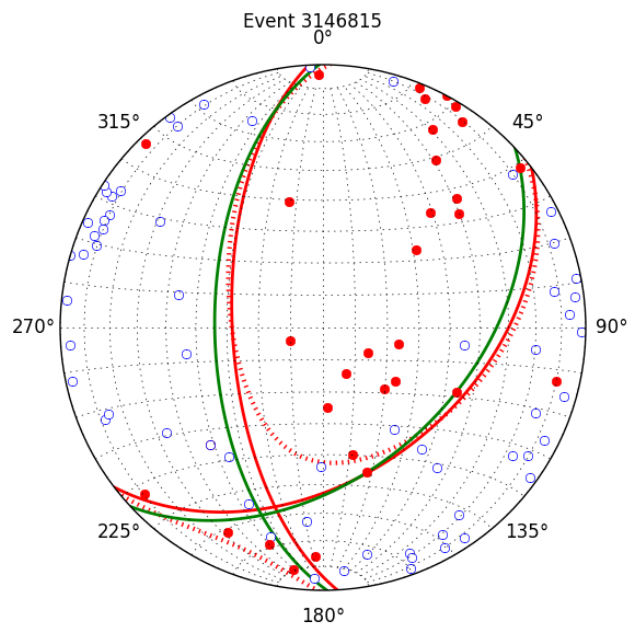

Fig. 7: For event 314681 from NorthRidge dataset. The green nodal line estimated by HASH and the solid red line is the optimal solution for the nodal lines derived from the SVC assuming a shear source. The dashed red line is the nodal line estimated by the SVC.

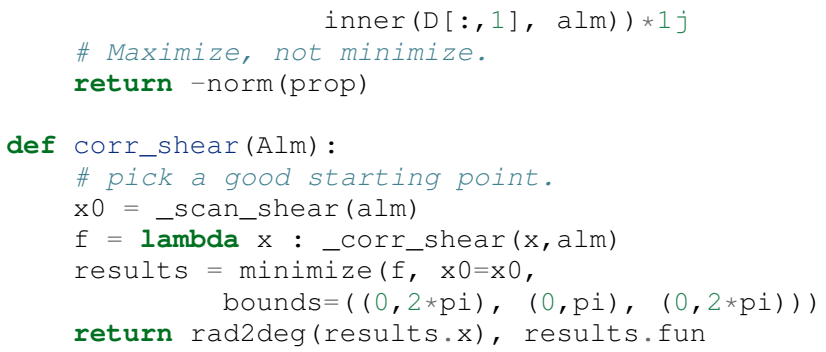

The function corr_shear shown in the code snippet implements the optimization of the above equation. The function WignerD2 implements the Wigner-D matrices defined in [Morrison], the variable "prop" is the projection of the learned solution onto the rotated template shear solution shown in Table 1, and Alm is the learned spectral content of the source. The initial guess is found scanning a coarse grid to find the best the quadrant with the highest initial correlation. This stops SciPy's default minimization [scipy] getting stuck in a local minima.

As an example, in Fig. 7 we show the classification results for the 3146815 event. The (dashed red) line shows the nodal line of the classifier function. The (solid red) line is the template shear solution, orientated by optimizing the correlation function, and the (solid green) line shows the preferred solution estimated by HASH.

\section{Discussion}

In Figures 6 and 7 we have shown examples of the classification and fault plane estimation methods. In this section we want to explore the robustness of the algorithm and try to gain some insight into the utility of the correlation functions.

The HASH program has an input (scsn.reverse) which identifies stations whose polarity was found to be erroneous in the past. These reversals are applied post facto to correct the input polarity data. We will use this feature to demonstrate an example where the support vector and least squares classifiers behave differently. In Fig 8 we give an example where we flipped the polarity of a single datum (indicated by the black arrow). The corresponding solutions are shown with (solid lines) and without (dashed lines) the benefit 


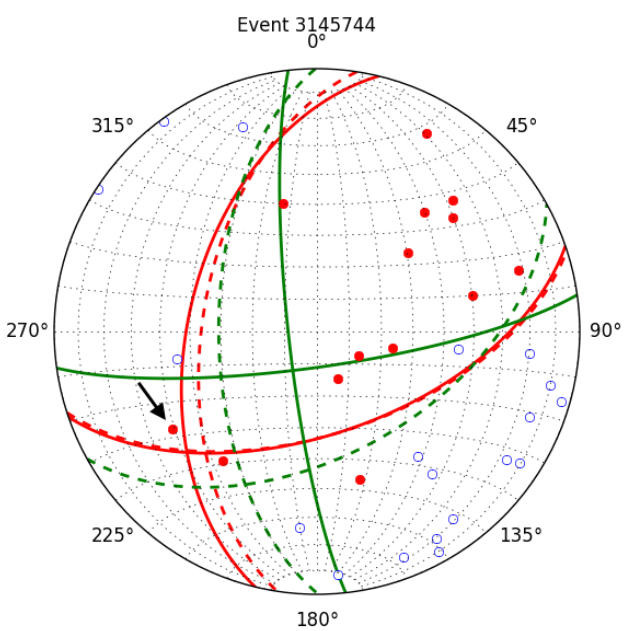

Fig. 8: For event 3145744 from the NorthRidge dataset. The color scheme for each subplot as in Fig. 7, the dashed lines are solutions without the station reversal being applied. The black arrow points to datum for which the polarity is flipped.

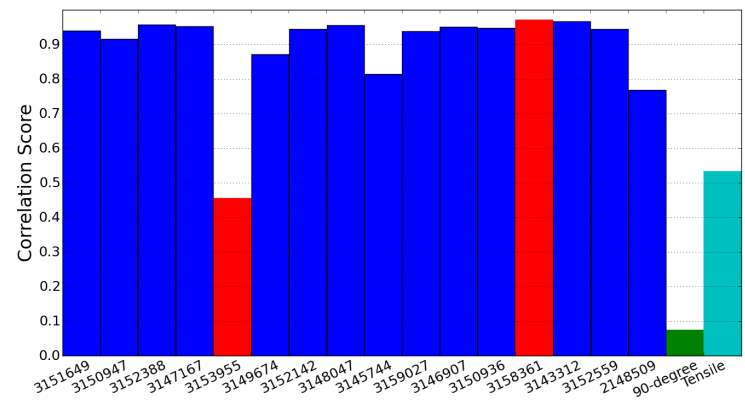

Fig. 9: The correlation score for each event in the Northridge dataset, comparing to event 3146815. (red) The events with maximum and minimum correlation score. (green) The correlation between 3146815 and itself rotated by 90-degrees strike, (cyan) the correlation between 3146815 and the tensile source found in Table 1.

of the polarity correction. The datum that was changed is close to the nodal line estimated by the SVC, which, given the soft thresholding, is forgiving of misclassification along its separating margin. The SVC solution for the nodal line is largely unchanged. On the other hand, the strategy of FPFIT is to minimize the overall rate of misclassification. And indeed, in each case, it finds the optimal solution on this basis. In fact, in terms of misclassified points, FPFIT outperforms the SVC classifier. But we would question whether minimizing the overall rate of misclassification is reasonable from an applied perspective. Consider that since the nodal line represents a point where the radiation pattern is changing sign, we expect that the signal to noise level will be smaller in this region. Conversely, from the point of view of the SVC, these are also the points that are most informative to the proper location of its separating margin. Indeed, many of the best quality picks far from the nodal lines will not influence the solution for the separating plane (recall dual coefficients can be zero). And it is reasonable that data of the correct class located far from the separating margin should not influence the solution. Looking at the problem from this perspective the solution of the SVC is more reasonable.
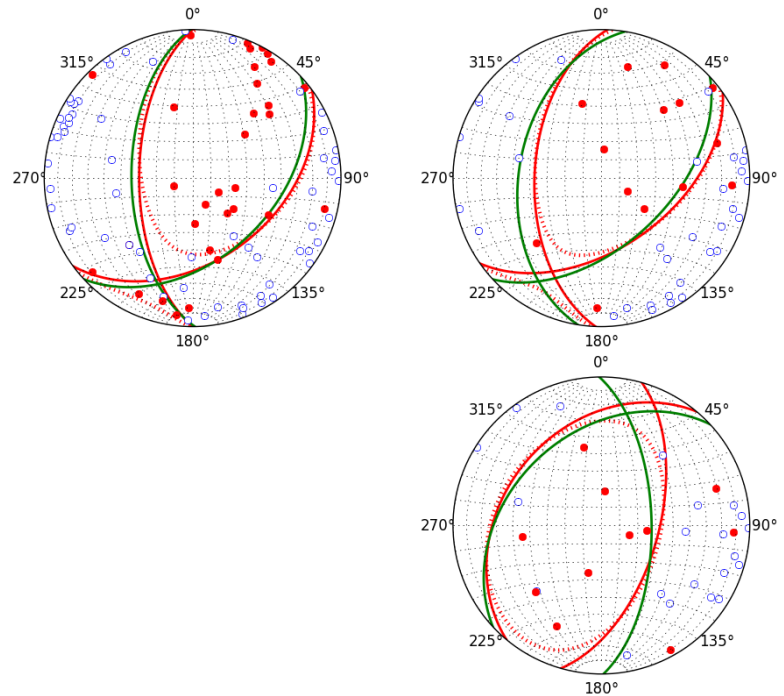

Fig. 10: The color scheme for each subplot as in Fig. 7. (top left) The solution for event 3146815 , (top right) the solution for events 3158361 and (bottom right) 3153955. Events 3158361 and 3153955 represent the maximum and minimum correlation score with event 3146815.

Finally, we derived a metric of similarity based on a correlation score Eq. 3. To provide an example of how we might use this correlation score, we take the event 3146815 , which has the largest number of data associate with it, and compute the correlation coefficient with each of the other events in the Northridge dataset. According to [HASH], the NorthRidge dataset we analyzed is expected to contain similar source mechanisms and certainly we see that the correlation score is high for the majority of the events. To test the sensitivity of the metric, we also compute the correlation between event 3146815 and itself rotated by 90degrees strike, and we see that this has low correlation, which we would expect.

In Fig. 10 we provide a visualization of the events with the highest (top right) and lowest (bottom right) correlation score comparing with event 3146815 (top left). The orientation of the nodal lines for event 3153955 , which has the lowest correlation score, indeed is qualitatively different than the solution for event 3146815. Qualitatively, we have demonstrated that the correlation score is a reasonable metric of similarity. Determining the actual statistical significance of the correlation score is left as future work.

\section{Conclusions}

We have presented a tool for classifying and comparing earthquake source mechanisms using tools from the scientific Python ecosystem. The important steps were to define the problem in terms of classification, which is solved robustly by the scikitlearn [sklearn] support vector classifier. We then used results from seismic source theory [Ben81] to derive a mapping between the input and feature spaces of the classification problem. Using the representation of the solution in the feature space, we derived a correlation coefficient.

This allowed us to generalize the earthquake classification to support both shear and tensile sources. As a particular example, we showed how maximizing correlation with the template shear solution could be used to estimate fault plane orientation. The key 
to efficiency here was to generate rotations in the feature space of the problem using Wigner's D matrices.

At each step along the way, we made a comparison with similar solutions obtained with the HASH algorithm [HASH], and found good general agreement. However, we argued that for this application, the optimization strategy of the SVC should prove more robust to misclassification than the least squares method.

Finally, we showed qualitatively, that the correlation coefficient provided a good metric for comparison between sources within the Northridge dataset. This technique has some promise as a tool for earthquake monitoring.

\section{Appendix}

The template solutions shown in Table 1 were derived from solutions tabulated in Table 4.4 of [Ben81]. Here, [Ben81] gives the solutions for the first P-wave arrival in terms of the Hansen vector $\mathrm{L}$ (in spherical polar coordinates) of the form,

$$
\vec{L}_{l m}(r, \theta, \phi)=\vec{\nabla} h_{l}^{2}(r) \tilde{Y}_{l m}(\theta, \phi),
$$

where " $h "$ is the spherical Hankel functions of a second kind. The amplitudes of the first break are required to be measured radially to the focal sphere, the projection of the Hansen vector radially is,

$$
\hat{r} \cdot \vec{L}_{l m}(r, \theta, \phi)=\frac{\partial}{\partial r} h_{l}^{2}(r) \tilde{Y}_{l m}(\theta, \phi) .
$$

The angular variation is given by the spherical harmonic function, up to an overall phase associated with radial component. Asymptotically (measurements are made far from the source), in this limit the Hankel functions tend to [Morse53],

$$
h_{l}^{2}(x)=\frac{1}{x}(i)^{l+1} \exp ^{-i x},
$$

which introduces a relative phase when collecting terms of different degree. We also note that the normalization of the spherical harmonics used in [Ben81] does not include the Cordon Shortley phase convention. Since we are using Wigner-D matrices to generate rotations, it is convenient to use that convention,

$$
\tilde{Y}_{l m}(\theta, \phi)=(-1)^{m} \sqrt{\frac{4 \pi(l+m) !}{(2 l+1)(l-m) !}} Y_{l m}(\theta, \phi) .
$$

The reference implementation ${ }^{6}$ includes its own sph_harm function to add this phase factor. With these adjustments, the amplitudes (up to an overall constant) for a common set of source mechanism, in terms of the spherical harmonics, are given in Table 1 .

\section{REFERENCES}

[Ben81]

[Aki02]

A. Ben-Menahem and S. J. Singh Seismic Waves and Sources Springer-Verlag New York Inc., 1981

[Morse53] M. Morse and F. Feshbach, Methods of theoretical physics Feschbach Publishing LLC, 1953

[HASH] J. L. Hardeback and P. M. Shearer, A New Method for Determining First-Motion Focal Mechanisms, Bulletin of the Seismological Socity of America, Vol. 92, pp 2264-2276, 2002

[FPFIT] Reasenberg, P., and D. Oppenheimer (1985). FPFIT, FPPLOT, and FPPAGE: FORTRAN computer programs for calculating and displaying earthquake faultplane solutions, U.S. Geol. Surv. Open-File Rept. 85-739, 109 Pp.
[Morrison]

M. A. Morrison and G. A. Parker, Australian Journal of Physics 40, 465 (1987).

[Scholkopf] B. Scholkopf and A. Smola, Learning with Kernels, The MIT Press, 2002

[sklearn] Fabian Pedregosa, Gaël Varoquaux, Alexandre Gramfort, Vincent Michel, Bertrand Thirion, Olivier Grisel, Mathieu Blondel, Peter Prettenhofer, Ron Weiss, Vincent Dubourg, Jake Vanderplas, Alexandre Passos, David Cournapeau, Matthieu Brucher, Matthieu Perrot, Édouard Duchesnay. Scikit-learn: Machine Learning in Python, Journal of Machine Learning Research, 12, 2825-2830 (2011)

[scipy] Stéfan van der Walt, S. Chris Colbert and Gaël Varoquaux. The NumPy Array: A Structure for Efficient Numerical Computation, Computing in Science \& Engineering, 13, 22-30 (2011), DOI:10.1109/MCSE.2011.37 (publisher link)

[mpl] John D. Hunter. Matplotlib: A 2D Graphics Environment, Computing in Science \& Engineering, 9, 90-95 (2007), DOI:10.1109/MCSE.2007.55

[ObsPy] M. Beyreuther, R. Barsch, L. Krischer, T. Megies, Y. Behr and J. Wassermann (2010), ObsPy: A Python Toolbox for Seismology, SRL, 81(3), 530-533, DOI: 10.1785/gssrl.81.3.530

[HashPy] hashpy, https://github.com/markcwill/hashpy, DOI: $10.5281 /$ zenodo. 9808

[mplstereonet] mplstereonet, https://pypi.python.org/pypi/mplstereonet 